\title{
Relativistic gyratons in asymptotically AdS spacetime
}

\author{
Valeri P. Frolov ${ }^{1, \text { 田 }}$ and Andrei Zelnikov ${ }^{1,2,2}$ 丹 \\ ${ }^{1}$ Theoretical Physics Institute, University of Alberta, Edmonton, AB, Canada, T6G 2J1 \\ ${ }^{2}$ Lebedev Physics Institute, Leninsky prospect 53, 119991, Moscow Russia \\ ${ }^{3}$ Asia Pacific Center for Theoretical Physics, Pohang 790-784, Korea
}

(Dated: November 13, 2017)

\begin{abstract}
We study the gravitational field of a spinning radiation beam-pulse (a gyraton) in a $D$-dimensional asymptotically AdS spacetime. It is shown that the Einstein equations for such a system reduce to a set of two linear equations in a $(D-2)$-dimensional space. By solving these equations we obtain a metric which is an exact solution of gravitational equations with the (negative) cosmological constant. The explicit metrics for $4 D$ and $5 D$ gyratons in asymptotically AdS spacetime are given and their properties are discussed.
\end{abstract}

PACS numbers: 04.70.Bw, 04.50.+h, 04.20.Jb

Alberta-Thy-11-05

\section{INTRODUCTION}

The gravitational field created by beams of radiation, and pulses of light has been studied intensively since a pioneer paper by Tolman [1] who found the solution of gravitational equations in linear approximation. The exact solutions of the Einstein equations for the pencil of light has been found by Peres [2, 3] and Bonnor [4]. The gravitational field of a spinning beam-pulse of finite duration, a gyraton, generalizes these solutions to the case when the beam-pulse carries an angular momentum [5, 6]. A typical example of a gyraton would be a pulse of a circular polarized light or a modulated beam of ultrarelativistic particles with a spin. The gravitational field of the gyraton is parametrized by a number of arbitrary functions of the retarded time $u$. These functions arise through the dependence on $u$ of the coefficients in mode expansion of the gravitational field. They describe profiles of the energy density and angular momenta distributions of the gyraton propagating in an asymptotically flat $D$-dimensional spacetime. The gyraton solutions in asymptotically flat spacetimes belong to a general class of pp-waves. In the limit of an infinitesimally short impulse and zero angular momentum the solutions describes a gravitational field of an ultrarelativistic particle - a gravitational shock wave [7].

In this paper we generalize results for gyratons in asymptotically flat spacetime [5, 6] to the case when a spacetime is asymptotically AdS. That is, we obtain exact solutions for the geometry of the gyraton propagating in an asymptotically AdS background. For zero angular momentum these solutions belong to the type of Siklos spacetimes [8] generalized to higher dimensions. In the limit of a $\delta(u)$-like impulse these solutions correspond to gravitational shock waves in AdS spacetime. Similar to shock waves in a flat spacetime the solutions can be derived using an infinite boost of the gravitational field of a

\footnotetext{
*Electronic address: frolov@phys.ualberta.ca
}

†Electronic address: zelnikov@phys.ualberta.ca point particle provided the energy of the particle is kept fixed [9, 10, 11] (the Penrose limit). Shock wave metrics can also be obtained from a global AdS space by cut-andpaste technique $9,12,13$. In the string theory gravitational shock waves propagating in a flat background got much attention since they do not receive $\alpha^{\prime}$ corrections [14, 15. This property can be generalized also to the case of gravitational shock waves propagating in AdS metrics [16]. The proof of this property is based on geometrical arguments, namely, on the fact that all scalar invariants constructed from the Riemann tensor and its derivatives are the same for pure AdS and for AdS shock wave solutions. In the present paper we prove that this geometrical property is also valid in more general case of the gyraton AdS spacetime.

The paper is organized as follows. Section $\amalg$ collects formulas for the gyraton metric in the asymptotically flat spacetime obtained earlier in [5, 6]. This is done in order to fix notations. These formulas are also used later when we are discussing the asymptotically flat space limit of the obtained gyraton metrics in the AdS spacetime. In Section III] a set of equations for a gyratons in the asymptotically AdS spacetime is derived. A general solution of these equations is obtained in Section IV Explicit metrics for gyratons in $4 D$ and $5 D$ asymptotically AdS spacetimes are obtained in the Sections $\nabla$ and $\nabla]$ respectively. Some properties of these solutions and their applications and generalizations are discussed in Section VII. Appendices contain expressions for the scalar and vector Green functions in the AdS spacetime, which are used in the main text.

\section{GYRATONS IN ASYMPTOTICALLY FLAT SPACETIME}

A gravitational field of a gyraton propagating in an asymptotically flat spacetime has been found recently in [5, 6]. It is described by the metric

$$
\begin{array}{r}
d s^{2}=-2 d u d v+d \mathbf{x}^{2}+\Phi d u^{2}+2(\mathbf{A}, d \mathbf{x}) d u, \\
u=x^{1}, \quad v=x^{2}, \quad \mathbf{x}=x^{a} .
\end{array}
$$


The functions $\Phi(u, \mathbf{x})$ and $\mathbf{A}(u, \mathbf{x})$ do not depend on $v$. The spatial part of the metric (11) in the $(D-$ 2)-dimensional hyperplane transverse to the direction of the motion of the gyraton is flat,

$$
d \mathbf{x}^{2}=\delta_{a b} d x^{a} d x^{b}=\sum_{a=3}^{D}\left(d x^{a}\right)^{2} .
$$

$l=l^{\mu} \partial_{\mu}=\partial_{v}$ is a null Killing vector. The metrics of this form are the most general D-dimensional null Brinkmann metrics [17] with flat transverse space, which sometimes are called $p p$-wave metrics 18. In what follows we assume that Latin indices $a, b, \ldots$ for the coordinates in the transverse plane run from 3 to $D$. We assume that the sum is taken over the repeated Latin indices and omit the summation symbol. We denote covariant derivatives with respect to the flat spatial metric $\delta_{a b}$ by colon, ():a.

The functions $\Phi$ and $A_{a}$ can be considered as a scalar and a vector field in the $(D-2)$-dimensional Euclidean space which depend also on an external parameter $u$. The metric (11) is invariant under the coordinate transformation

$$
v \rightarrow v+\lambda\left(u, x^{a}\right),
$$

provided the functions $\Phi$ and $A_{a}$ transform as follows

$$
A_{a} \rightarrow A_{a}-\lambda_{, a}, \Phi \rightarrow \Phi-2 \lambda_{, u} .
$$

We shall also use the following notation

$$
F_{a b}=\partial_{a} A_{b}-\partial_{b} A_{a}
$$

for the antisymmetric tensor in the $(D-2)$-plane. This tensor is evidently invariant under the transformation (3).

The nonzero components of the Ricci tensor for the metric (11) are 6]

$$
\begin{aligned}
R_{u a} & =\frac{1}{2} F_{a b}: b \\
R_{u u} & =-\frac{1}{2} \Phi_{: a}^{: a}+\frac{1}{4} F_{a b} F^{a b}+\partial_{u}\left(A_{a}^{: a}\right) .
\end{aligned}
$$

Thus the Einstein equations reduce to the following two sets of equations in $(D-2)$-dimensional flat space

$$
\begin{aligned}
& F_{a b}: b=J_{a}, \\
& \Phi_{: a}^{a}=-J+\frac{1}{2} F_{a b} F^{a b}+2 \partial_{u}\left(A_{a}^{: a}\right),
\end{aligned}
$$

where

$$
J_{a}=\kappa T_{u a}, \quad J=\kappa\left[T_{u u}-\frac{1}{D-2} g_{u u} T\right] .
$$

Here $\kappa=16 \pi G$ and $G$ is the $D$-dimensional gravitational coupling constant.

The first set of equations (5) formally coincides with the Euclidean Maxwell equations in $D-2$-dimensional Euclidean space (magnetostatics), $J_{a}$ playing the role of the current. The second equation (6) is similar to the equation for the electric potential with the only difference that besides the charge distribution $J$ it contains an extra source proportional to $\mathbf{F}^{2}$.

To solve the equations (5)-(6) one should first find a vector potential from the linear equation (5), and then substitute the obtained solution to the right hand-side of (6). It is convenient to split $\Phi$ into two parts, the first part being a solution for the source $J$, and the second one being a solution for the distributed "charge" $J_{F}=-\frac{1}{2} \mathbf{F}^{2}$

$$
\begin{aligned}
& \Phi=\varphi+\psi, \\
& \varphi_{: a}^{a}-2 \partial_{u}\left(A_{a}^{: a}\right)=-J, \quad \psi_{: a}^{: a}=\frac{1}{2} F_{a b} F^{a b} .
\end{aligned}
$$

Note that the combination $\Phi_{: a}^{: a}-2 \partial_{u}\left(A_{a}: a\right)$ remains invariant under the gauge transformation (3). Therefore, the equations (5), (6), (8), and (9) are gauge invariant.

The source terms $J$ and $J_{a}$ vanish outside the position of the gyraton. Solutions obtained in [5, 6] describe the gravitational field of the gyraton in the limit when its transverse size goes to zero. Though it is possible to find an exact solution of the Einstein equations for an arbitrary source of finite size, it makes sense to consider first point-like distributions in the transverse space. Let us emphasized that in the general case the solution (9) is only formal and may not have a well-defined sense. The reason is that for a point-like current, $F_{a b}$ has a singularity at $\mathbf{x}=0$. If one considers this singular function as a distribution, one needs to define what is the meaning of $F_{a b} F^{a b}$ in (9). This problem does not exist for a distributed source (gyraton). At some small distance outside the point-like source the vacuum solution of the Einstein equations provides a description of the problem in question. At the position of the source this vacuum solution can be stitched to the metric created by gyraton with a generic distribution of the energy density and angular momentum ( see a discussion of the problem at the end of Chapter II of the paper [ 6$]$ ). In our analysis of the gyratons in the asymptotically AdS spacetime we assume that the source terms are locally the same as in the asymptotically flat case. One can reformulate this condition by requiring that the local properties of the gravitational field near $\mathbf{x}=0$ are the same in both cases.

\section{GYRATONS IN ASYMPTOTICALLY ADS SPACETIME}

Now consider a gyraton propagating in the Ddimensional asymptotically AdS background.

It is well known that a pure AdS spacetime is conformal to the Minkowski one. Let us choose one of the spatial coordinates, say $x^{3} \equiv z$, and consider the following metric

$$
d \bar{s}^{2}=\bar{g}_{\mu \nu} d x^{\mu} d x^{\nu}=\frac{L^{2}}{z^{2}}\left[-2 d u d v+d \mathbf{x}^{2}\right] .
$$


It is easy to show that this metric has constant curvature and obey the Einstein equations

$$
\bar{R}_{\mu \nu}-\frac{1}{2} \bar{R} \bar{g}_{\mu \nu}=-\Lambda \bar{g}_{\mu \nu},
$$

where $\Lambda=-(D-1)(D-2) /\left(2 L^{2}\right)$. The constant $L$ in (10) is the radius of the curvature of the AdS world. It is worthwhile to mention, that in the metric (10) the coordinate $z$ play a special role, and by using the conformal factor depending on $z$ one might expect that the symmetry of the metric (10) would be less than the symmetry of the original flat metric. It does not happen. The spacetime (10) remains homogeneous and isotopic, but instead of the Poincare group its isometry group is $S O(D, 1)$. In the solution (10) the point $z=0$ corresponds to the spatial infinity and $z=\infty$ is the horizon defined for the set of observers sitting at rest at constant $z$.

As the ansatz for the gyraton metric in the asymptotically AdS spacetime we use the following expression

$$
\begin{aligned}
d s^{2} & =\frac{L^{2}}{z^{2}}\left[-2 d u d v+d \mathbf{x}^{2}\right. \\
& \left.+\Phi(u, \mathbf{x}) d u^{2}+2(\mathbf{A}(u, \mathbf{x}), d \mathbf{x}) d u\right] .
\end{aligned}
$$

Here again $z=x^{3}$ is one of the spatial coordinates. In the absence of the gyraton $\Phi=\mathbf{A}=0$ and the metric reduces to the pure AdS metric. This property would be preserved asymptotically if one assumes that both functions $\Phi(u, \mathbf{x})$ and $\mathbf{A}(u, \mathbf{x})$ vanish at the infinity of the transverse space. In what follows we assume that this condition is satisfied. Note also that in the limit $L \rightarrow \infty$, while the location of the gyraton is kept near $z_{0} \rightarrow L$ and $\left[\left(z-z_{0}\right)^{2}+\left(\mathbf{x}-\mathbf{x}_{0}\right)^{2}\right] / L^{2} \rightarrow 0$, one gets the asymptotically flat gyraton geometry.

Before discussing solutions of the Einstein equations of the form (12) note that this metric has the following property: All local scalar invariants constructed from the metric, the Riemann tensor and its covariant derivatives are exactly the same as those for the pure AdS spacetime (10). This property is valid off-shell, i.e., the metric does not need to be a solution of the Einstein equations. The proof of this statement is given in Appendix $\mathrm{A}$ This is a generalization to the case of relativistic gyratons of the statement by Horowitz and Itzhaki [16] which has been given in application to gravitational shock waves in AdS.

Now let us return to the dynamics of the gyraton spacetime. Substituting the ansatz (12) into the Einstein equations

$$
R_{\alpha \beta}-\frac{1}{2} R g_{\alpha \beta}+\Lambda g_{\alpha \beta}=8 \pi T_{\alpha \beta}
$$

and using the same notations (2),(41), and (7) as in asymptotically flat case, we obtain again two nontrivial equations

$$
\begin{aligned}
F_{a b}: b & -\frac{D-2}{z} F_{a z}=J_{a}, \\
\Phi_{: a}^{: a}-\frac{1}{2} F_{a b} F^{a b} & -2 \partial_{u}\left(A_{a}^{: a}\right) \\
& -\frac{D-2}{z}\left(\partial_{z} \Phi-2 \partial_{u} A_{z}\right)=-J .
\end{aligned}
$$

Here (): $a$ denotes the covariant derivatives with respect to the flat metric in the transverse space.

\section{SOLVING THE EINSTEIN EQUATIONS}

\section{A. Magnetostatics in AdS space}

The analogy of (13) with the "magnetostatic" Maxwell equations (5) can be made more precise if we rewrite (13) as the Maxwell equations in a fiducial $\mathrm{N}$-dimensional Euclidean AdS space (B1). To do this let us introduce a fiducial Euclidean AdS metric $\tilde{g}_{A B}$

$$
\tilde{g}_{A B}=\frac{L^{2}}{z^{2}} \delta_{A B}, \quad A, B=(3, \ldots, N+2) .
$$

For simplicity we put the radius parameter $L=1$. It is not difficult to restore proper dimensionality later. Let us denote the covariant derivatives in AdS metric (15) by semicolon and covariant derivatives in flat $\mathrm{N}$-dimensional metric by a vertical bar. Then one has

$$
F_{A B} ; B=z^{2}\left(F_{A B} \mid B-\frac{N-4}{z} F_{A z}\right) .
$$

One can see that the equation (13) is identical to the Maxwell equations in $N=D+2$ dimensional Euclidean AdS space and reads

$$
\begin{gathered}
F_{A B} ; B=\tilde{J}_{A}, \quad \tilde{J}_{A}=z^{2} J_{A}, \\
F_{A B}=\partial_{A} A_{B}-\partial_{B} A_{A}, \\
A_{A}=\left(A_{a}, 0,0,0,0\right), \quad J_{A}=\left(J_{a}, 0,0,0,0\right) .
\end{gathered}
$$

We call this space fiducial. The current $J_{A}$ and the vector potential $A_{A}$ depend only on $D-2$ coordinates $x^{a}$ and the retarded tine $u$. To obtain a solution for $A_{A}$ one needs to know the Green function for vector field in Euclidean AdS space

$$
A_{A}(x)=\int d^{N} x^{\prime} \sqrt{\tilde{g}^{\prime}} G_{A B^{\prime}}\left(x, x^{\prime}\right) \tilde{J}^{B^{\prime}}\left(x^{\prime}\right) .
$$

The vector Green function $G_{A B^{\prime}}$ in the pure AdS space is well known 21, 23. We present the explicit expression for it in Appendix [C] 


\section{B. Massless scalar field in AdS space}

Similarly to the vector field case we represent (14) in the form of the scalar field equation in the fiducial higher dimensional Euclidean AdS space (B1).

$$
\bar{g}_{A B}=\frac{L^{2}}{z^{2}} \delta_{A B}, \quad A, B=(3, \ldots, D+1, D+2) .
$$

One has $A_{A}=\left(A_{a}, 0,0\right)$,

$$
\begin{aligned}
& \Phi_{; A}^{; A}=z^{2}\left(\Phi_{\mid A}^{\mid A}-\frac{D-2}{z} \Phi_{, z}\right)=z^{2}\left(\Phi_{: a}^{: a}-\frac{D-2}{z} \Phi_{, z}\right), \\
& A_{A}^{; A}=z^{2}\left(A_{A}^{\mid A}-\frac{D-2}{z} A_{z}\right)=z^{2}\left(A_{a}^{: a}-\frac{D-2}{z} A_{z}\right), \\
& F_{A B} F^{A B}=z^{4} F_{a b} F^{a b} .
\end{aligned}
$$

For the scalar equations the dimensionality of the fiducial space $N=D$ is chosen so that these equations reproduce (14). Thus we can rewrite equation (14) for the metric of the gyraton as an equation for the functions $\Phi$ defined in the pure Euclidean AdS geometry with the proper number of dimensions $(N=D)$. The scalar equation then reads

$$
\Phi_{; A}^{; A}=-z^{2} J+\frac{1}{2 z^{2}} F_{A B} F^{A B}+2 \partial_{u}\left(A_{A}^{; A}\right) .
$$

A solution of this problem expressed in terms of the scalar Green function (B4) in the D-dimensional Euclidean AdS space is

$$
\begin{aligned}
\Phi & =\varphi+\psi \\
\varphi & =\int d^{D} x \sqrt{\bar{g}^{\prime}} G\left(x, x^{\prime}\right) \bar{J}\left(x^{\prime}\right) \\
\psi & =\int d^{D} x \sqrt{\bar{g}^{\prime}} G\left(x, x^{\prime}\right) \bar{J}_{F}\left(x^{\prime}\right), \\
\bar{J} & =\left[z^{2} J-2 \partial_{u}\left(A_{A}^{; A}\right)\right], \quad \bar{J}_{F}=-\frac{1}{2 z^{2}} F_{A B} F^{A B} .
\end{aligned}
$$

\section{V. $4 D$ GYRATONS}

Let us consider a special case of a gyraton in $4 D$ AdS. Its metric has the form

$d s^{2}=\frac{-2 d u d v+d z^{2}+d x^{2}+\Phi d u^{2}+2\left(A_{z} d z+A_{x} d x\right) d u}{z^{2}}$.

The spatial components $J_{a}$ of the vector current are assumed to be localized at $x=x_{0}$ and to have the same structure as in asymptotically flat case [5, 6]

$$
J_{a}=\frac{\kappa}{2} j(u) z^{2} \epsilon_{a b} \partial_{b}\left[\delta\left(z-z_{0}\right) \delta\left(x-x_{0}\right)\right] .
$$

Here $\epsilon_{a b}$ is the 2D Levi-Civita symbol and the total angular momentum of the source is given by $\mathcal{J}=\int d u j(u)$. The current satisfies the conservation law

$$
\partial^{a} J_{a}-\frac{2}{z} J_{z}=0
$$

We look for the vector potential in the form

$$
A_{a}=z^{2} \epsilon_{a b} \partial_{b} \sigma(z, x)
$$

which, evidently, satisfies the gauge condition

$$
\partial^{a} A_{a}-\frac{2}{z} A_{z}=0
$$

The two-dimensional field strength is given by the relation

$$
F_{a b}=\partial_{a} A_{b}-\partial_{b} A_{a}=-\epsilon_{a b} \partial_{c}\left(z^{2} \partial^{c} \sigma\right)
$$

We restrict ourselves to the case when $F_{a b}$ decreases at spatial infinity, then from the equations (13) one gets

$$
\frac{1}{z^{2}} \partial_{a}\left(z^{2} \partial^{a} \sigma\right)=-\frac{\kappa}{2} j(u) \delta\left(z-z_{0}\right) \delta\left(x-x_{0}\right)
$$

Looking at (25) and (26) one can see that in four dimensions due to the Maxwell equations $F_{a b}$, vanishes everywhere outside the location of the gyraton [25], while $A_{a}$ is nontrivial.

The solution for $\sigma$ can be easily found. It reads

$$
\begin{aligned}
\sigma & =\frac{\kappa}{8 \pi} j(u) \frac{1}{z z_{0}} \ln \frac{\mathcal{U}}{\mathcal{U}+2} \\
& =\frac{\kappa}{8 \pi} j(u) \frac{1}{z z_{0}} \ln \frac{\left(z-z_{0}\right)^{2}+\left(x-x_{0}\right)^{2}}{\left(z+z_{0}\right)^{2}+\left(x-x_{0}\right)^{2}}
\end{aligned}
$$

where

$$
\mathcal{U}=\frac{\left(z-z_{0}\right)^{2}+\left(x-x_{0}\right)^{2}}{2 z z_{0}}=2 \frac{r_{-}^{2}}{r_{+}^{2}-r_{-}^{2}}
$$

and

$$
r_{ \pm}=\sqrt{\left(z \pm z_{0}\right)^{2}+\left(x-x_{0}\right)^{2}}
$$

Thus we obtain

$$
\begin{aligned}
A_{a} & =\frac{\kappa}{8 \pi} j(u) z^{2} \epsilon_{a b} \partial_{b}\left[\frac{1}{z z_{0}} \ln \frac{\left(z-z_{0}\right)^{2}+\left(x-x_{0}\right)^{2}}{\left(z+z_{0}\right)^{2}+\left(x-x_{0}\right)^{2}}\right] \\
& =\frac{\kappa}{\pi} j(u) z^{2} \epsilon_{a b} \partial_{b}\left[\frac{1}{r_{+}^{2}-r_{-}^{2}} \ln \frac{r_{-}}{r_{+}}\right]
\end{aligned}
$$

Now consider the scalar equation (14)

$$
\partial^{a} \partial_{a} \Phi-\frac{2}{z} \partial_{z} \Phi=-J+\frac{1}{2} F_{a b} F^{a b}
$$

where

$$
J=-\kappa \sqrt{2} \varepsilon(u) z^{2} \delta\left(z-z_{0}\right) \delta\left(x-x_{0}\right)
$$

A solution for $\Phi$ can be found by using the method discussed in the previous section. In fact we use (22) by introducing two extra dimensions $\mathbf{y}=\left(y^{1}, y^{2}\right)$ and using 
scalar Green functions (B5) given in Appendix B For $F_{a b}=0$ one has 26$]$

$$
\begin{aligned}
\Phi & =-\kappa \sqrt{2} \varepsilon(u) \int d \mathbf{y} G_{4}\left(z, x, \mathbf{y} ; z_{0}, x_{0}, 0\right) \\
& =-\frac{\kappa \sqrt{2}}{4 \pi} \varepsilon(u) z z_{0}\left[(\mathcal{U}+1) \ln \frac{\mathcal{U}}{\mathcal{U}+2}+2\right] \\
& =-\frac{\kappa \sqrt{2}}{8 \pi} \varepsilon(u)\left[\left(r_{+}^{2}+r_{-}^{2}\right) \ln \frac{r_{-}}{r_{+}}+r_{+}^{2}-r_{-}^{2}\right] .
\end{aligned}
$$

Here functions $\mathcal{U}$ and $r_{ \pm}$are defined by (28) and (29).

Note that the four dimensional gyraton is a special case, because the equations for $F_{a b}$ assume that it vanishes outside the location of the gyraton. Therefore, locally the vector function $A_{a}$ can be put to zero using a proper coordinate transformation (3). In this gauge the metric acquires the Siklos [8] form. However, one can not make $A_{a}$ to be zero globally, since the gauge invariant contour integral $\oint A_{a}(u, \mathbf{x}) d x^{a}$ around the position of the gyraton is proportional to the angular momentum density $j(u)$ which is non-zero. This is why we prefer to present the vector potential $A_{a}$ in the form (30), which is analogous to the vector potential of the Bohm-Aharonov flux. Let us emphasize that, for higher dimensions $D \geq 5$ the tensor $F_{a b}$ is non-trivial anyway.

\section{VI. $5 D$ GYRATONS}

In five dimensions AdS gyraton metric reads

$$
d s^{2}=\frac{-2 d u d v+\left(d x^{a}\right)^{2}+\Phi d u^{2}+2 A_{a} d x^{a} d u}{z^{2}},
$$

where $x^{a}=\left(z, x^{3}, x^{4}\right)$. The gyraton current has a form

$$
J_{a}=\frac{\kappa}{2} z^{3} j(u) \epsilon_{a b c} n^{c} \partial_{b}\left[\delta^{3}\left(x^{a}-x_{0}^{a}\right)\right] .
$$

It is parametrized by a unit 3-vector $n^{c}$ which points in the direction of the magnetic dipole. Without loss of generality one can write

$$
n^{c}=\delta_{z}^{c} \cos \eta+\delta_{3}^{c} \sin \eta, \quad \eta=\text { const. }
$$

This current satisfies the conservation law

$$
J_{a}^{: a}-\frac{3}{z} J_{z}=0 .
$$

Let us consider the simplest case when the current is in the $\left(x^{3}, x^{4}\right)$ plane, i.e., dipole moment is directed along $z$-axis, $n^{c}=(1,0,0)$. Then

$$
J_{a}=\frac{\kappa}{2} z^{3} j(u) \epsilon_{a b z} \partial_{b}\left[\delta^{3}\left(x^{a}-x_{0}^{a}\right)\right] .
$$

In the Lorentz gauge

$$
A_{a}^{: a}-\frac{3}{z} A_{z}=0,
$$

the vector potential can be written in the form

$$
A_{a}=\epsilon_{a b z} \partial_{b}\left[\sigma\left(x^{c}\right)\right] .
$$

The corresponding Maxwell tensor takes the form

$$
\begin{aligned}
& F_{a b}=\epsilon_{a b c} H^{c}, \quad H^{c}=\frac{1}{2} \epsilon^{c e f} F_{e f}, \\
& H^{c}=-\delta_{z}^{c} \partial_{b} \partial^{b} \sigma+\partial_{z} \partial^{c} \sigma, \\
& F_{a b}=-\epsilon_{a b z} \partial^{c} \partial_{c} \sigma+\epsilon_{a b c} \partial_{z} \partial^{c} \sigma, \\
& F_{a b}: b-\frac{3}{z} F_{a z}=-\epsilon_{a b z} \partial^{b}\left[\partial_{c} \partial^{c} \sigma-\frac{3}{z} \partial_{z} \sigma\right] .
\end{aligned}
$$

From this we obtain the following equation for $\sigma$

$$
\partial_{c} \partial^{c} \sigma-\frac{3}{z} \partial_{z} \sigma=-\frac{\kappa}{2} z^{3} j(u) \delta^{3}\left(x^{a}-x_{0}^{a}\right),
$$

which coincides with the scalar field equation in $5 D \mathrm{AdS}$ spacetime. Using the scalar Green functions (B5) we get

$$
\begin{aligned}
\sigma & =-\frac{\kappa}{4 \pi} j(u) z z_{0} \frac{[\mathcal{U}+1-\sqrt{\mathcal{U}(\mathcal{U}+2)}]^{2}}{2 \sqrt{\mathcal{U}(\mathcal{U}+2)}} \\
& =-\frac{\kappa}{64 \pi} j(u) \frac{\left(r_{+}-r_{-}\right)^{4}}{r_{+} r_{-}} .
\end{aligned}
$$

Here

$$
\mathcal{U}=\frac{\left(z-z_{0}\right)^{2}+\mathbf{x}^{2}}{2 z z_{0}}, \quad r_{ \pm}=\sqrt{\left(z \pm z_{0}\right)^{2}+\mathbf{x}^{2}},
$$

and $\mathbf{x}^{2}=\left(x^{3}-x_{0}^{3}\right)^{2}+\left(x^{4}-x_{0}^{4}\right)^{2}$.

In the vicinity of the current, i.e, when $r_{-} \rightarrow 0$ one gets

$$
\sigma \rightarrow-\frac{\kappa}{8 \pi} j(u) \frac{z_{0}^{3}}{r_{-}} .
$$

Contrarily to $4 \mathrm{D}$ case, the Maxwell tensor $F_{a b}$ in five dimensions does not vanish outside the source. The corresponding "magnetic" field $H^{c}$ is

$$
H^{c}=\left(\partial^{c}-\frac{3}{z} \delta_{z}^{c}\right) \partial_{z} \sigma+\frac{\kappa}{2} j(u) \delta_{z}^{c} z^{3} \delta\left(z-z_{0}\right) \delta(\mathbf{x}) .
$$

The equation for $\Phi$ involves the current $J$ (see (7)) and

$$
J_{F}=-\frac{1}{2} F_{a b} F^{a b}=-H^{c} H_{c} .
$$

The local terms, originating from $\delta$-function, have meaning of proper energy of the gyraton and can be combined into the redefined energy parameter $\mathcal{E}$ of the source. So, the nontrivial contribution of angular momenta to the $\Phi$ component of the gyraton metric comes from the first term in (38).

The solution for the $g_{u u}$ component of the metric follows the same lines as that of $4 \mathrm{D}$ case

$$
\begin{aligned}
\Phi & =\varphi+\psi, \\
\varphi_{: a} a & -\frac{3}{z} \partial_{z} \varphi=-J, \\
\psi_{: a}^{: a}-\frac{3}{z} \partial_{z} \psi & =-J_{F} .
\end{aligned}
$$


The scalar current is

$$
J=-\kappa \sqrt{2} z^{3} \varepsilon(u) \delta\left(x^{a}-x_{0}^{a}\right) .
$$

Because the equation for $\varphi$ is exactly of the same type as (35), we can write down the answer without further calculations

$$
\begin{aligned}
\varphi & =\frac{\kappa \sqrt{2}}{2 \pi} \varepsilon(u) z z_{0} \frac{[\mathcal{U}+1-\sqrt{\mathcal{U}(\mathcal{U}+2)}]^{2}}{2 \sqrt{\mathcal{U}(\mathcal{U}+2)}} \\
& =\frac{\kappa \sqrt{2}}{32 \pi} \varepsilon(u) \frac{\left(r_{+}-r_{-}\right)^{4}}{r_{+} r_{-}} .
\end{aligned}
$$

Using the same Green function (B5) we can write the solution for $\psi$

$$
\psi=\int d z^{\prime} d \mathbf{x}^{\prime} \mathbf{G}\left(z, \mathbf{x} ; z^{\prime} \mathbf{x}^{\prime}\right) \frac{1}{z^{\prime 3}} J_{F}^{\prime} .
$$

Here

$$
\begin{gathered}
\mathbf{G}\left(z, \mathbf{x} ; z^{\prime} \mathbf{x}^{\prime}\right)=-\frac{1}{8 \pi \sqrt{\left(\left(z-z^{\prime}\right)^{2}+\Delta \mathbf{x}^{2}\right)\left(\left(z+z^{\prime}\right)^{2}+\Delta \mathbf{x}^{2}\right)}} \\
\times\left[z^{2}+z^{\prime 2}+\Delta \mathbf{x}^{2}-\sqrt{\left(\left(z-z^{\prime}\right)^{2}+\Delta \mathbf{x}^{2}\right)\left(\left(z+z^{\prime}\right)^{2}+\mathbf{x}^{2}\right)}\right]^{2}, \\
\Delta \mathbf{x}^{2}=\left(\mathbf{x}-\mathbf{x}^{\prime}\right)^{2}=\left(x^{3}-x^{\prime 3}\right)^{2}+\left(x^{4}-x^{\prime 4}\right)^{2},
\end{gathered}
$$

and $J_{F}^{\prime}=-H^{\prime c} H_{c}^{\prime}$ is a function of $\left(z^{\prime}, z_{0}, \mathbf{x}^{\prime}\right)$ and $H_{c}$ is defined by (38).

\section{SUMMARY AND DISCUSSIONS}

The main result of this paper is the generalization of the gyraton solutions [5, 6] to asymptotically AdS spacetimes. These metrics describe the gravitational field of ultrarelativistic beam pulses with non-zero angular momentum propagating in the AdS spacetime. We demonstrate how the method proposed in [5, 6] can be generalized to solve the Einstein equations for the spacetime which is asymptotically AdS. The corresponding solutions contain a number of arbitrary functions of $u$ describing distributions of the energy density and angular momenta of the beam pulse. As special examples we discuss the gyraton AdS metrics in 4 and 5 dimensions in detail. In the absence of angular momentum the 4D AdSgyraton solutions reduce to the Siklos spacetimes [8]. In the absence of rotation and for a $\delta$-function profile of the energy density, the obtained solutions coincide with AdS shock waves metric 19.

The gyraton geometry in asymptotically flat spacetimes has the property that all scalar invariants constructed from the curvature and its covariant derivatives are zero [6]. It is this property, that in the case of shock waves made it possible to conclude that quantum and $\alpha^{\prime}$ corrections to the metric are zero 14, 15]. We demonstrate that a similar property is valid for the gyraton AdS metrics. Namely, all scalar invariants constructed from the Riemann tensor and its covariant derivatives are the same both for the asymptotically AdS gyraton metric and for exact AdS spacetime. It was shown earlier [20] that in the string theory $\alpha^{\prime}$ corrections do not modify AdS solution. Thus, according to the geometrical approach arguments [16] these corrections, probably, should not modify the asymptotically AdS gyraton metric as well.

It should be emphasized that we focused on the solutions outside the region occupied by a gyraton. To obtain a total solution one needs to solve the interior problem inside the region occupied by the gyraton and to glue this solution with an exterior metric. Solutions for $4 \mathrm{D}$ and $5 \mathrm{D}$ AdS gyratons presented in the paper (which formally has a singularity at $\mathbf{x}=0$ ) generalize special solutions discussed in [5, [6].

The obtained AdS gyraton solutions can be used for study of the mini black hole production in the collision of two ultrarelativistic particles with spin moving in the AdS space. It would be also interesting to analyze these solutions in relation with the AdS-CFT correspondence. In particular, a gyraton may have a complex structure which is encoded in its gravitational field. According to the AdS-CFT correspondence the asymptotic of this metric at the AdS space infinity must be sufficient to obtain the complete information about the gyraton structure. It might be possible since all the multipole moments of the field in the AdS do not fall of faster at infinity 24]. It is interesting to discuss this mechanism in more details.

\section{Acknowledgments}

The authors are grateful to Werner Israel for stimulating discussions and remarks. This work was supported by the Natural Sciences and Engineering Research Council of Canada, by the Killam Trust and in part by APCTP.

\section{APPENDIX A: CURVATURE INVARIANTS}

\section{Curvature}

Let us demonstrate that the metric (12) has the following property: All local invariants constructed from the metric, the Riemann tensor and its covariant derivatives are exactly the same as those for the pure AdS spacetime (10). This property is valid off-shell, i.e., the metric does not need to be a solution of the Einstein equations.

To prove this we write the gyraton metric (12) in the form

$$
g_{\alpha \beta}=\bar{g}_{\alpha \beta}+2 l_{(\alpha} a_{\beta)},
$$

where $\bar{g}_{\alpha \beta}$ is the AdS metric (10) and the vectors $l_{\alpha}$ and $a_{\alpha}$ (in the coordinates adopted in (10) and (12)) have the 
components

$$
\begin{aligned}
l_{u} & =-\frac{L^{2}}{z^{2}}, \quad l_{v}=l_{a}=0, \\
a_{u} & =\frac{1}{2} \Phi, \quad a_{v}=0, \quad a_{a}=A_{a}(u, \mathbf{x}) .
\end{aligned}
$$

In what follows we shall use the gyraton metric $g_{\alpha \beta}$ to operate with indices. For example, $a^{\alpha}=g^{\alpha \beta} a_{\beta}$ and $l^{\alpha}=$ $g^{\alpha \beta} l_{\beta}$. In particular, one has

$$
l^{\alpha}=\delta_{v}^{\alpha}, \quad l^{\alpha} a_{\alpha}=0 .
$$

It is easy to see that $\mathbf{l}=l^{\alpha} \partial_{\alpha}$ is the Killing vector in the both metrics (10) and (12). This vector obey the following properties

$$
\begin{aligned}
l^{\epsilon} l_{\epsilon} & =0, \quad l_{; \epsilon}^{\epsilon}=0, \\
l_{\alpha ; \beta} & =2 l_{[\alpha} \kappa_{\beta]}, \quad l^{\epsilon} \kappa_{\epsilon}=0,
\end{aligned}
$$

where $\kappa_{\alpha}=-\nabla_{\alpha} \ln z$. Here semicolon denotes the covariant derivative with respect to the gyraton metric. We use notations $l_{(\alpha} a_{\beta)}=\frac{1}{2}\left(l_{\alpha} a_{\beta}+l_{\beta} a_{\alpha}\right)$ and $l_{[\alpha} \kappa_{\beta]}=$ $\frac{1}{2}\left(l_{\alpha} \kappa_{\beta}-l_{\beta} \kappa_{\alpha}\right)$.

Straightforward calculations show that the scalar curvature for the metric (12) is constant $R=-\frac{D(D-1)}{L^{2}}$. The Riemann tensor for the gyraton metric can be written as the sum

$$
R_{\alpha \beta \mu \nu}=\hat{R}_{\alpha \beta \mu \nu}+r_{\alpha \beta \mu \nu} .
$$

Here the constant curvature part reads

$$
\hat{R}_{\alpha \beta \mu \nu}=\left[g_{\alpha \mu} g_{\beta \nu}-g_{\alpha \nu} g_{\beta \mu}\right] R .
$$

Let us express the Riemann tensor of the AdS metric $\bar{g}_{\alpha \beta}$ in terms of the gyraton metric $g_{\alpha \beta}$. We have

$$
\bar{g}_{\alpha \beta}=g_{\alpha \beta}-2 l_{(\alpha} a_{\beta)} .
$$

The inverse AdS metric, being expressed in terms of the gyraton metric, has a form

$$
\bar{g}^{\alpha \beta}=g^{\alpha \beta}+2 l^{(\alpha} a^{\beta)}+l^{\alpha} l^{\beta} a^{\epsilon} a_{\epsilon} .
$$

Straightforward calculation of the Christoffel symbols gives

$$
\bar{\Gamma}_{\alpha \beta}^{\mu}=\Gamma_{\alpha \beta}^{\mu}-\gamma_{\alpha \beta}^{\mu},
$$

where

$$
\begin{aligned}
\gamma_{\alpha \beta}^{\mu} & =l^{\mu} a_{(\alpha ; \beta)}+2 a_{(\alpha} l_{; \beta)}^{\mu}+l_{(\alpha} F_{\beta)}{ }^{\mu} \\
& +2 l^{\mu} a^{\epsilon} l_{\epsilon ;(\alpha} a_{\beta)}+l^{\mu} l_{(\alpha} F_{\beta) \epsilon} a^{\epsilon} .
\end{aligned}
$$

Here $F_{\alpha \beta}=\partial_{\alpha} a_{\beta}-\partial_{\beta} a_{\alpha}$. The 'spatial' components of this tensor, $F_{a b}$, coincide with (4), and $F_{\alpha \beta} l^{\beta}=0$. It is easy to check that the tensor $\gamma_{\alpha \beta}^{\mu}$ obeys the relations

$$
\gamma_{\alpha \beta}^{\mu} l_{\mu}=\gamma_{\alpha \beta}^{\mu} l^{\alpha}=\gamma_{\alpha \beta}^{\mu} l^{\beta}=0 .
$$

Using (A12) and the property (A6) one can show that

$$
\begin{gathered}
\gamma_{\alpha \beta}^{\mu}=l^{\mu} p_{\alpha \beta}+l_{(\alpha} q_{\beta)}{ }^{\mu}, \\
l^{\alpha} p_{\alpha \beta}=l^{\beta} q_{\beta}{ }^{\mu}=l_{\mu} q_{\beta}{ }^{\mu}=0 .
\end{gathered}
$$

We call a tensor to be aligned to the the vector $l_{\alpha}$ if it can be written as a sum of terms, where each term contains as a factor at least one vector $l_{\alpha}$. The tensor $\gamma_{\alpha \beta}^{\mu}$ is aligned to the vector $l_{\alpha}$.

Substituting the decomposition (A11) into the definition of the Riemann tensor

$$
R_{\nu \alpha \beta}^{\mu}=\partial_{\alpha} \Gamma_{\nu \beta}^{\mu}-\partial_{\beta} \Gamma_{\nu \alpha}^{\mu}+\Gamma_{\epsilon \alpha}^{\mu} \Gamma_{\nu \beta}^{\epsilon}-\Gamma_{\epsilon \beta}^{\mu} \Gamma_{\nu \alpha}^{\epsilon}
$$

we obtain

$\bar{R}_{\nu \alpha \beta}^{\mu}=R_{\nu \alpha \beta}^{\mu}-\nabla_{\alpha} \gamma_{\nu \beta}^{\mu}+\nabla_{\beta} \gamma_{\nu \alpha}^{\mu}+\gamma_{\epsilon \alpha}^{\mu} \gamma_{\nu \beta}^{\epsilon}-\gamma_{\epsilon \beta}^{\mu} \gamma_{\nu \alpha}^{\epsilon}$.

It's easy to see that all terms containing $\gamma_{\nu \beta}^{\mu}$ and $\nabla_{\alpha} \gamma_{\nu \beta}^{\mu}$ are aligned with $l_{\alpha}$ owing to (A13) and (A6). The difference of the Riemann tensors with all indices in the lower position has the same property because lowing index on the left hand-side with (A9) results to additional term $-l^{\mu} a_{\epsilon} R_{\nu \alpha \beta}^{\epsilon}$ on the right hand-side, which is orthogonal to $l_{\alpha}$. Therefore, the difference $r_{\mu \nu \alpha \beta}$ of the Riemann tensors

$$
\bar{R}_{\mu \nu \alpha \beta}=R_{\mu \nu \alpha \beta}-r_{\mu \nu \alpha \beta}
$$

is aligned with the vector $l_{\alpha}$ and has the form

$$
r_{\mu \nu \alpha \beta}=l_{[\mu} K_{\nu][\alpha \beta]}+l_{[\alpha} K_{\beta][\mu \nu]},
$$

where the tensor $K_{\mu[\alpha \beta]}$ is orthogonal to $l^{\alpha}$

$$
l^{\mu} K_{\mu[\alpha \beta]}=l^{\alpha} K_{\mu[\alpha \beta]}=0 .
$$

\section{Curvature invariants}

At first we consider scalar invariants constructed from the curvature but which do not contain covariant derivatives. Scalar invariants, constructed from powers of the curvature $R_{\alpha \beta \mu \nu}$ involve the powers of $\hat{R}_{\alpha \beta \mu \nu}$, (A8), constructed from the gyraton metric, and the powers of $r_{\alpha \beta \mu \nu}$. But the powers of $r_{\alpha \beta \mu \nu}$ contain at least one $l_{\alpha}$ which must be contracted either with another $l_{\alpha}$, or with $a_{\alpha}$ and $K_{\alpha \mu \nu}$. In all these cases the contraction gives zero. Therefore, only the powers of $\hat{R}_{\alpha \beta \mu \nu}$ may survive a contraction over all indexes. Hence, such invariants calculated for the gyraton metric are identical to those of the exact AdS geometry.

Now consider scalar invariants containing covariant derivatives of the curvature with respect to the gyraton metric $g_{\alpha \beta}$. The derivatives of the constant curvature part in the decomposition (A7) vanish identically. So, there remain only terms with derivatives acting on $r_{\alpha \beta \mu \nu}$ given by (A16). Since $r_{\alpha \beta \mu \nu}$ is aligned with the vector $\mathbf{l}$, 
terms which enter a given scalar invariant always contain either $\mathbf{l}$ or its covariant derivatives. Besides these terms, the scalar invariant may also include terms constructed from $a_{\alpha}, \kappa_{\alpha}, K_{\alpha \mu \nu}$ and their covariant derivatives. In the general case the scalar invariant is a linear combination of products of such terms. For each of the product one can define the total number of covariant derivatives which enter the product. Suppose that $n$ is the largest of these numbers, than we denote the corresponding scalar invariant by $S_{n}$. We call $n$ an order of the invariant.

In what follows we shall use the property that $\mathbf{l}$ is the Killing vector. Denote by $T_{\alpha . .}^{\beta \ldots}$ a tensor constructed from $a_{\alpha}, \kappa_{\alpha}, K_{\alpha \mu \nu}$ and their covariant derivatives. Then

$$
\mathcal{L}_{1} T_{\alpha \ldots}^{\beta \ldots}=0,
$$

or using the definition of the Lie derivative

$$
l^{\mu} \nabla_{\mu} T_{\alpha \ldots}^{\beta \ldots}=\nabla_{\mu} l^{\alpha} T_{\mu \ldots}^{\beta \ldots}+\ldots-\nabla_{\beta} l^{\mu} T_{\alpha \ldots}^{\mu \ldots}-\ldots .
$$

Consider a scalar invariant $S_{n}$. We describe now operations which allows one to transform identically $S_{n}$ into a 'canonical' form. Each of these operations either keeps the order $n$ the same or reduces it.

Operation 1. If in an invariant $S_{n}$ there exists a covariant derivative acting in $\mathbf{l}$, one can use (A6) to exclude it. By repeating this procedure one can always transform $S_{n}$ into the form $S_{n^{\prime}}^{\prime}$ without derivatives of $\mathbf{l}$. It is evident that $n^{\prime} \leq n$.

Operation 2. Consider an invariant $S_{n}$ which does not contain derivatives of $\mathbf{l}$. Since $\mathbf{l}^{2}=0$, the vector index of $l^{\alpha}$ must be contracted either with the index of $a_{\alpha}$, $\kappa_{\alpha}, K_{\alpha \mu \nu}$, or with one of the covariant derivatives. In the former case, the corresponding term is of the form $l^{\alpha} \nabla \ldots \nabla p_{\ldots \alpha \ldots}$, where $p_{\ldots \alpha \ldots}$ is one of the tensors $a_{\alpha}$, $\kappa_{\alpha}, K_{\alpha \mu \nu}$. Using the relation

$$
l_{\alpha} \nabla_{\beta}(\ldots)=\nabla_{\beta}\left[l_{\alpha}(\ldots)\right]-2 l_{[\alpha} \kappa_{\beta]}(\ldots),
$$

one can 'move' $\mathbf{l}$ through covariant derivatives. By repeating this procedure and using (A6) one finally tranform $S_{n}$ into the form

$$
S_{n}=\nabla \ldots \nabla\left(l^{\alpha} p_{\ldots \alpha} \ldots\right)+S_{n-1} .
$$

Since $l^{\alpha} p_{\ldots \alpha \ldots}=0$ as a final result of this procedure one can exclude terms containing $l^{\alpha} \nabla \ldots \nabla p_{\ldots \alpha} \ldots$ and decrease the order $n$. By using again (if necessary) the Operation 1 one can transform the obtained expression $S_{n-1}$ into the form without the derivatives of $\mathbf{l}$.

Operation 3. Consider now a term where the index of $\mathbf{l}$ is contracted with the index of a covariant derivative

$$
l^{\alpha} \ldots \nabla_{\alpha}(\ldots) .
$$

Using (A19) one can transform the corresponding invariant $S_{n}$ (without increasing its order) into a form

$$
\ldots l^{\alpha} \nabla_{\alpha}(\ldots) .
$$

By using (A18) and (A6) one can decrease the number of derivative in this expression. As the result, one reduces $S_{n}$ to $S_{n-1}$. By using (if necessary) the Operation 1 one can transform the obtained expression $S_{n-1}$ into the form without the derivatives of $\mathbf{l}$.

By repeating the operations 2 and 3 one finally arrives to an invariant of the zero order in derivatives. But all zero order invariants necessarily contain as factors the contraction of $l^{\epsilon}$ with $a_{\epsilon}, \kappa_{\epsilon}$, and $K_{\epsilon \mu \nu}$, which are zero. Thus, only invariants which did not contain derivatives may not vanish. But, as was shown the beginning of this subsection, these invariants for AdS and AdS-gyraton metrics are identical. Therefore, all scalar invariants for the gyraton in the asymptotically AdS spacetime and scalar invariants of exact AdS spacetime coincide. It should be emphasized that we do not use the fact that $\Lambda$ is negative. Thus the same result is also valid in the asymptotically de Sitter spacetime.

\section{APPENDIX B: SCALAR GREEN FUNCTION IN ADS}

The Euclidean propagator for a massless scalar field is the solution to the equation

$$
\square G\left(x, x^{\prime}\right)=-\delta\left(x, x^{\prime}\right), \quad x^{A}=\left(z, x^{4}, \ldots, x^{N+2}\right) .
$$

Here we enumerated coordinates starting with $x^{3}=z$, rather than $x^{1}$ in order to distinguish this artificial Euclidean AdS from the physical spacetime. The Euclidean $A d S_{N}$ metric with a unit radius $L=1$ reads

$$
d s^{2}=\frac{1}{z^{2}}\left[(d z)^{2}+\left(d x^{4}\right)^{2}+\cdots+\left(d x^{N+2}\right)^{2}\right] .
$$

The geodesic distance $\mu\left(x, x^{\prime}\right)$ in this geometry is

$$
\begin{gathered}
\cosh \left(\mu\left(x, x^{\prime}\right)\right)=1+U\left(x, x^{\prime}\right), \\
U=\frac{\delta_{A B}\left(x^{A}-x^{\prime A}\right)\left(x^{B}-x^{\prime B}\right)}{2 z z^{\prime}} .
\end{gathered}
$$

It obeys the equation

$$
\mu_{A} \mu^{A}=1, \quad U_{A} U^{A}=U(U+2) .
$$

Because AdS is highly symmetrical space all necessary physical quantities may be expressed in terms of only one biscalar function (see, e.g., 21] $) \mu\left(x, x^{\prime}\right)$ or $U \equiv$ $U_{N}\left(x, x^{\prime}\right)$. Thus for the Green function in even $(N=2 k)$ and odd $(N=2 k+1)$ number of dimensions we obtain accordingly [22]

$$
\begin{aligned}
G_{2 k}\left(x, x^{\prime}\right) & =-\frac{1}{(2 \pi)^{k}}\left(-\frac{\partial}{\partial U}\right)^{k-1} Q_{k-1}(U+1), \\
G_{2 k+1}\left(x, x^{\prime}\right) & =-\frac{1}{(2 \pi)^{k}}\left(-\frac{\partial}{\partial U}\right)^{k-1} \\
& \times \frac{[U+1-\sqrt{U(U+2)}]^{k}}{2 \sqrt{U(U+2)}} .
\end{aligned}
$$


Here $Q_{k-1}(U+1)$ is the Legendre function and $U=$ $U_{N}\left(x, x^{\prime}\right)$.

For the problem in question the source on the right hand-side of the (21) depends on the retarded time $u$ and $(D-2)$ spatial coordinates $\left(z, x^{4}, \ldots, x^{D}\right)$. Therefore, the solution for the scalar potential $\Phi$ will require integration over all unphysical extra dimensions. For our purpose we need to know an integral of the scalar Green function over two extra coordinates (e.g., $x^{N+1}$ and $x^{N+2}$ ).

$$
\int d x^{N+1} d x^{N+2} G\left(U_{N}\right)=2 \pi z z^{\prime} \int_{U_{N-2}}^{\infty} d U_{N} G\left(U_{N}\right) .
$$

This property trivially follows from the relation

$$
\begin{aligned}
U_{N} & =U_{N-2}+\frac{l^{2}}{2 z z^{\prime}}, \\
l^{2} & =\left(x^{N+1}-x^{\prime+1}\right)^{2}+\left(x^{N+2}-x^{N+2}\right)^{2} .
\end{aligned}
$$

The Legendre function of integer index can be expressed in terms of elementary functions. For example explicit expressions for the Green functions from 2 till 6 dimensions are

$$
\begin{aligned}
& G_{2}=\frac{1}{4 \pi} \ln \left(\frac{U}{U+2}\right) \\
& G_{3}=-\frac{1}{4 \pi}\left[\frac{U+1}{\sqrt{U(U+2)}}-1\right] \\
& G_{4}=-\frac{1}{8 \pi^{2}}\left[\ln \left(\frac{U}{U+2}\right)+\frac{1}{U}+\frac{1}{U+2}\right] \\
& G_{5}=-\frac{1}{8 \pi^{2}}\left[2+\frac{1-3 U-6 U^{2}-2 U^{3}}{(U(U+2))^{3 / 2}}\right] \\
& G_{6}=\frac{1}{16 \pi^{3}}\left[3 \ln \left(\frac{U}{U+2}\right)+\frac{2(U+1)\left(3 U^{2}+6 U-2\right)}{U^{2}(U+2)^{2}}\right] .
\end{aligned}
$$

\section{APPENDIX C: GREEN FUNCTION FOR A MASSLESS VECTOR FIELD IN ADS}

Consider a Green function $G_{A B^{\prime}}\left(x, x^{\prime}\right)$ for massless vector fields in Euclidean AdS space. This propagator satisfies the equation

$$
\nabla^{A} \nabla_{[A} G_{B] B^{\prime}}=-g_{B B^{\prime}} \delta\left(x, x^{\prime}\right)+\partial_{B^{\prime}} \Lambda_{B}\left(x, x^{\prime}\right)
$$

and can be represented in the form

$$
G_{A B^{\prime}}\left(x, x^{\prime}\right)=-\left(\partial_{A} \partial_{B^{\prime}} U\right) F(U)+\partial_{A} \partial_{B^{\prime}} S(U) .
$$

Here $F(U)$ describes the propagation of the physical components of $A_{A}$ while $\Lambda_{B}$ and $S(U)$ are gauge artifacts and can be discarded [21, 23].

$$
\begin{aligned}
F(U)=C_{N}[ & U(2+U)]^{1-N / 2}, \quad C_{N}=\frac{\Gamma\left(\frac{N}{2}-1\right)}{(4 \pi)^{N / 2} .} \\
\partial_{A} \partial_{B^{\prime}} U=- & \frac{1}{z z^{\prime}}\left[\delta_{A B^{\prime}}-U \delta_{A z} \delta_{z B^{\prime}}\right. \\
& \left.+\frac{1}{z}\left(x-x^{\prime}\right)_{A} \delta_{z B^{\prime}}-\frac{1}{z^{\prime}}\left(x-x^{\prime}\right)_{B^{\prime}} \delta_{A z} \cdot\right] .
\end{aligned}
$$

The vector potential is defined as

$$
A_{A}(x)=\int d^{N} x^{\prime} \sqrt{g^{\prime}} G_{A B^{\prime}}\left(x, x^{\prime}\right) J^{B^{\prime}}\left(x^{\prime}\right) .
$$

Similar to the scalar field case the current $J^{B^{\prime}}$ doesn't depend on some coordinates $x^{k}$ and doesn't have components in these directions. Then integration over these coordinates reduces to the integration of the scalar function $F(U)$.

Integral of $F(U)$ over two "redundant" coordinates gives

$$
\begin{aligned}
\int d x^{N+1} d x^{N+2} F\left(U_{N}\right)=2 \pi z z^{\prime} \int_{U_{N-2}}^{\infty} d U_{N} F\left(U_{N}\right) \\
\int_{U_{N-2}}^{\infty} d U_{N} F\left(U_{N}\right)=C_{N} \frac{\left(U_{N-2}\right)^{3-N}}{N-3} \\
\times F\left(N-3, \frac{N}{2}-1 ; N-2 ;-\frac{2}{U_{N-2}}\right) .
\end{aligned}
$$

For example, for $N=4$

$$
\frac{1}{C_{4}} \int_{U_{2}}^{\infty} d U_{4} F\left(U_{4}\right)=-\frac{1}{2} \ln \frac{U_{2}}{U_{2}+2} ;
$$

for $N=5$

$$
\frac{1}{C_{5}} \int_{U_{3}}^{\infty} d U_{5} F\left(U_{5}\right)=\frac{U_{3}+1}{\sqrt{U_{3}\left(U_{3}+2\right)}}-1
$$

for $N=6$

$$
\frac{1}{C_{6}} \int_{U_{4}}^{\infty} d U_{6} F\left(U_{6}\right)=\frac{1}{4}\left[\ln \frac{U_{4}}{U_{4}+2}+\frac{1}{U_{4}}+\frac{1}{U_{4}+2}\right] ;
$$

for $N=7$

$$
\begin{aligned}
\frac{1}{C_{7}} \int_{U_{5}}^{\infty} d U_{7} F\left(U_{7}\right)= & -\frac{2}{3} \frac{U_{5}+1}{\sqrt{U_{5}\left(U_{5}+2\right)}} \\
& +\frac{2}{3}+\frac{1}{3} \frac{U_{5}+1}{\left[U_{5}\left(U_{5}+2\right)\right]^{\frac{3}{2}}} .
\end{aligned}
$$

The integral of the function $F(U)$ over four "redundant" coordinates gives

$$
\begin{aligned}
& \int d x^{N-1} d x^{N} d x^{N+1} d x^{N+2} F\left(U_{N}\right) \\
& =\left(2 \pi z z^{\prime}\right)^{2} \int_{U_{N-4}}^{\infty} d U_{N-2} \int_{U_{N-2}}^{\infty} d U_{N} F\left(U_{N}\right) \\
& =C_{N} \frac{\left(2 \pi z z^{\prime}\right)^{2} U_{N-4}^{4-N}}{(N-3)(N-4)} F\left(N-4, \frac{N}{2}-1 ; N-2 ;-\frac{2}{U_{N-4}}\right) .
\end{aligned}
$$

For example, one has for $N=5$

$\frac{1}{C_{5}} \int_{U_{1}}^{\infty} d U_{1} \int_{U_{3}}^{\infty} d U_{3} F\left(U_{5}\right)=-\sqrt{U_{1}\left(U_{1}+2\right)}+U+1 ;$ 
for $N=6$

$\frac{1}{C_{6}} \int_{U_{2}}^{\infty} d U_{4} \int_{U_{4}}^{\infty} d U_{6} F\left(U_{6}\right)=\frac{1}{4}\left(U_{2}+1\right) \ln \left(\frac{U_{2}}{U_{2}+2}\right)+\frac{1}{2} ;$

for $N=7$

$\frac{1}{C_{7}} \int_{U_{3}}^{\infty} d U_{5} \int_{U_{5}}^{\infty} d U_{7} F\left(U_{7}\right)=\frac{2}{3}\left[\sqrt{U_{3}\left(U_{3}+2\right)}-U-1\right.$

$\left.+\frac{1}{\sqrt{U_{3}\left(U_{3}+2\right)}}\right]$.

[1] R. C. Tolman, Relativity, Thermodynamics, and Cosmology, p.272, Oxford, Clarendon Press (1934).

[2] A. Peres, Phys. Rev. Lett. 3, 571 (1959).

[3] A. Peres, Phys. Rev. 118, 1105 (1960).

[4] W. B. Bonnor, Commun. Math. Phys. 13, 163 (1969).

[5] V.P. Frolov and D.V. Fursaev, Phys.Rev. D71, 104034 (2005); hep-th/0504027

[6] V.P. Frolov, W. Israel, and A. Zelnikov, hep-th/0506001

[7] P.C. Aichelburg and R.U. Sexl, Gen.Rel.Grav. 2 303 (1971).

[8] S.T.C. Siklos, in Galaxies, Axisymmetric Systems and Relativity, edited by M.A.H. MacCallum (Cambridge University Press, Cambridge, England, 1985);

J. Podolsky, Class.Quant.Grav. 15719 (1998).

[9] T. Dray and G.'t Hooft, Nucl.Phys.B 253173 (1985); Class.Quant.Grav.3 825 (1986).

[10] V. Ferrari, P. Pendenza, G. Veneziano, Gen.Rel.Grav. 20 1185 (1988); H. de Vega, N. Sanchez, Nucl.Phys.B 317 706 (1989).

[11] C. Barrabes, P.A. Hogan Phys.Rev. D 640044022 (2001).

[12] K. Sfetsos, Nucl.Phys.B 436721 (1995).

[13] N. Kaloper, Phys.Rev. D71 $086003 \quad$ (2005); hep-th/0502035

[14] G.T. Horowitz, A.R. Steif, Phys.Rev.Lett. 64260 (1990).

[15] D. Amati, C. Klimcik, Phys.Lett. B219 443 (1989).

[16] G.T. Horowitz, N. Itzhaki, JHEP 9902:010 (1999); hep-th/9901012
[17] H.W. Brinkmann, Math.Ann. 94119 (1925).

[18] A.A. Tseytlin, Class.Quant.Grav. 122365 (1995); M. Blau, M. O‘Loughlin, G. Papadopoulos and A.A. Tseytlin, Nucl.Phys.B 67357 (2003).

[19] J. Podolsky, Class.Quant.Grav. 153229 (1998).

[20] R. Kallosh, A. Rajaraman, Phys.Rev. D58 125003 (1998); hep-th/9805041

[21] B. Allen, T. Jacobson, Commun.Math.Phys. 103669 (1986).

[22] U.H. Danielsson, E. Keski-Vakkuri and M. Kruczenski, JHEP 9901002 (1999); hep-th/9812007

[23] E. D'Hoker, D.Z. Freedman, S.D. Mathur, A. Matusis, L. Rastelli, Nucl.Phys.B 562330 (1999); hep-th/9902042

[24] g.t. Horowitz, V.E. Hubeny, JHEP 0010:027 (2000); hep-th/0009051

[25] Generically one could add to $F_{a b}$ the solution of the homogeneous equation $\sim z^{2} \epsilon_{a b}$, but this term would violate our asymptotic requirements

[26] For an extended gyraton $F_{a b}$ does not vanish in a region occupied by the gyraton, so that $\mathbf{F}^{2}$-term in (31) gives a non-trivial contribution to $\Phi$. This contribution depends on the size of the gyraton as well as on its structure. In the simplest case in the limit of a small size gyratons this contribution results in the renormalization of the energy density $\varepsilon(u)$. For details see [6]. 